\title{
The Role of Nanoengineered Wettability in Phase Change Heat Transfer
}

\author{
Antonio Luis Moreira \\ Universidade de Lisboa, Portugal \\ Av. Rovisco Pais 1, 1049-001 Lisboa, Portugal
}

When a liquid becomes in contact with a solid surface, such in a heat transfer device, the interfacial transport of mass, momentum and energy is determined by the ability of the liquid to maintain contact with the surface, resulting from intermolecular interactions when the two are brought together. With this in mind, recent progresses in micro-/nanofabrication and coating technologies have enabled the development of smart engineered interfaces. On the solid side of the interface, surface structuring techniques allow tailoring special lyophobic and lyophilic mixed surfaces with great potential for miniaturized heat transfer devices and are currently driving a renewable research interest for both, experimental and theoretical, studies in multi-scale transport phenomena. Also, the addition of nano-sized particles (1-100 nm) to the cooling fluid alter the free energy on the liquid side of the interface and, therefore, the wetting behaviour.

In this context, laboratory studies have been conducted aimed at contributing to the development of advanced miniaturized cooling systems. In this lecture, the interfacial transport phenomena in pool boiling, single and multimicrochannel flows and droplet cooling are addressed from the perspective of their potential to develop smart engineered interfaces capable of enhancing the performance of practical heat transfer devices. 